\title{
Hypermethylation of BDNF and SST Genes in the Orbital Frontal Cortex of Older Individuals: A Putative Mechanism for Declining Gene Expression with Age
}

\author{
Brandon C McKinney', Chien-Wei Lin², Hyunjung Oh ${ }^{1,3}$, George C Tseng ${ }^{2}$, David A Lewis ${ }^{1,3}$ and \\ Etienne Sibille ${ }^{*, 1,3,4}$
}

'Department of Psychiatry, University of Pittsburgh Medical School, Pittsburgh, PA, USA; ${ }^{2}$ Department of Biostatistics, University of Pittsburgh, Pittsburgh, PA, USA; ${ }^{3}$ Center for Neuroscience, University of Pittsburgh, Pittsburgh, PA, USA; ${ }^{4}$ Campbell Family Mental Health Research Institute of CAMH, Departments of Psychiatry, Pharmacology and Toxicology, University of Toronto, Toronto, ON, Canada

\begin{abstract}
Expression of brain-derived neurotrophic factor (BDNF) and somatostatin (SST) mRNAs in the brain decreases progressively and robustly with age, and lower BDNF and SST expression in the brain has been observed in many brain disorders. BDNF is known to regulate SST expression; however, the mechanisms underlying decreased expression of both genes are not understood. DNA methylation (DNAm) is an attractive candidate mechanism. To investigate the contribution of DNAm to the age-related decline in BDNF and SST expression, the Illumina Infinium HumanMethylation450 Beadchip Array was used to quantify DNAm of BDNF (26 CpG loci) and SST (9 CpG loci) in the orbital frontal cortices of postmortem brains from 22 younger (age $<42$ years) and 22 older individuals (age $>60$ years) with known agedependent BDNF and SST expression differences. Relative to the younger individuals, 10 of the 26 CpG loci in BDNF and 8 of the 9 CpG loci in SST were significantly hypermethylated in the older individuals. DNAm in BDNF exons/promoters I, II, and IV negatively correlated with BDNF expression ( $r=-0.37, p<0.05 ; r=-0.40, p<0.05 ; r=-0.24, p=0.07$ ), and DNAm in SST 5' UTR and first exon/intron negatively correlated with SST expression $(r=-0.48, p<0.0 I ; r=-0.63, p<0.00 \mathrm{I})$, respectively. An expanded set of BDNF- and GABArelated genes exhibited similar age-related changes in DNAm and correlation with gene expression. These results suggest that DNAm may be a proximal mechanism for decreased expression of BDNF, SST, and other BDNF- and GABA-related genes with brain aging and, by extension, for brain disorders in which their expression is decreased.
\end{abstract}

Neuropsychopharmacology (20I5) 40, 2604-26I3; doi:I0.I038/npp.20 I5.I07; published online I3 May 2015

\section{INTRODUCTION}

As the population 'grays', the burden of brain aging and of brain disorders for which age is a risk factor will grow without advances in prevention and treatment. Such advances depend on understanding the neurobiology underlying brain aging and late-life brain disorders. Previously, we proposed one putative neurobiological mechanism for latelife brain disorders when we described the 'age-by-disease hypothesis' which posits that these disorders are, in part, the result of changes in the expression of disease-related genes in the brain that occur naturally during brain aging (McKinney et al, 2012a; McKinney and Sibille, 2012b). Supporting this hypothesis is the observation that a consistent subset of genes undergoes age-dependent changes in expression, and this subset overlaps extensively with those that exhibit altered

*Correspondence: Dr E Sibille, Centre for Addiction and Mental Health (CAMH), 250 College Street, Room 134, Toronto, ON M5T IR8, Canada, Tel: + | 416535850।, ext 3657l,

E-mail: Etienne.sibille@camh.ca

Received 3 December 2014; revised 20 March 2015; accepted 8 April 2015; accepted article preview online 16 April 2015 expression in multiple brain disorders. Indeed, about a third of the genes that exhibit age-dependent changes in expression are altered in a range of brain disorders (eg, bipolar disorder, major depression, schizophrenia, Alzheimer's disease, Parkinson's disease, and Huntington's disease, amyotrophic lateral sclerosis) (Glorioso et al, 2011). Not only do many of the genes implicated in these diseases exhibit age-related changes in expression but also the direction of the change that occurs with age is usually in the direction thought to promote disease.

Brain-derived neurotrophic factor (BDNF) and somatostatin (SST) are genes whose expression is consistent with the 'age-by-disease hypothesis.' That is, reduced BDNF and SST expression is observed in multiple brain disorders (Autry and Monteggia, 2012; Guilloux et al, 2012; Lin and Sibille, 2013; Tapia-Arancibia et al, 2008; Tripp et al, 2011; Zuccato and Cattaneo, 2009), and their expression progressively and robustly declines in the brain with age (Erraji-Benchekroun et al, 2005; Glorioso et al, 2011; Hayashi et al, 1997; Keleshian et al, 2013; Webster et al, 2002). The mechanism of decline, however, is not understood. DNA methylation (DNAm), the covalent addition of a methyl group to a 
cytosine nucleotide in DNA, is a candidate mechanism given (1) that changes in DNAm near gene regulatory regions affect the expression of that gene (Baubec and Schubeler, 2014) and (2) that the abundance and pattern of DNAm changes with age (Fraga and Esteller, 2007). At least one study suggests DNAm as a mechanism for age-related changes in the expression of BDNF in the brain (Keleshian et al, 2013). Understanding how BDNF and SST expression declines with age may shed light on the mechanisms that are active in brain aging more broadly as well as the brain disorders with which their decreased expression has been associated.

We previously demonstrated that low SST expression in the brain is partly downstream from low BDNF expression (Guilloux et al, 2012; Tripp et al, 2012) and others have shown BDNF to be regulated by DNAm (Ikegame et al, 2013). Thus we hypothesized that DNAm is a mechanism contributing to age-related decreases in BDNF and SST expression. We predicted BDNF would be hypermethylated in older individuals, that BDNF methylation would negatively correlate with BDNF expression, and that SST methylation would be independent of age, consistent with the observation that low SST expression is downstream of low BDNF expression.

\section{MATERIALS AND METHODS}

\section{Postmortem Brains}

Brains were recovered during routine autopsies conducted at the Allegheny Country Medical Examiner's Office, Pittsburgh, PA following informed consent from the next-of-kin. A committee of clinicians made consensus DSM-IV diagnoses, or the absence thereof, based on clinical records and structured interviews with surviving relatives. Subjects with neurological disease or gross neuropathology were excluded from the study. The right hemisphere of each brain was cut and immediately frozen/stored at $-80^{\circ} \mathrm{C}$. Samples containing all six cortical layers, but excluding the adjacent white matter, were harvested from cryostat sections at the anatomical level corresponding to the orbital frontal cortex (OFC), specifically Brodmann's Areas 11 and 47. All procedures were approved by the University of
Pittsburgh Committee for the Oversight of Research and Clinical Trials Involving the Dead and the Institutional Review Board for Biomedical Research.

\section{Gene Expression}

Gene expression data from a previously published study were used for cohort selection and correlation with DNAm. Detailed methods are described elsewhere (Seney et al, 2013). Briefly, total RNA from OFC samples adjacent to those used in the present study were processed for analysis on the Affymetrix GeneChip Human Gene 1.1. ST (Affymetrix, Santa Clara, CA, USA).

\section{Cohort Membership}

Twenty-two individuals $\leqslant 42$ years of age and 22 individuals $\geqslant 60$ years of age comprised the younger and older cohorts, respectively. None met criteria for DSM-IV diagnoses. We selected the individuals with the highest (younger cohort) or lowest (older cohort) BDNF and SST mRNA levels (Supplementary Table S1). Cohorts did not differ with respect to postmortem interval (PMI), pH, RNA integrity number (RIN; Agilent Bioanalyzer, Santa Clara, California, USA), sex, or race (Table 1 and Supplementary Table S1).

\section{DNA Preparation and Bisulfite Conversion}

Genomic DNA (gDNA) was isolated using the AllPrep DNA/RNA/Protein Mini Kit (Qiagen, Valencia, CA, USA). Purity was assessed by NanoDrop 1000 (Thermo Scientific, Willmington, DE, USA), and bisulfite conversion was performed using the Zymo EZ-96 DNA Methylation Kit (Zymo Research, Irvine, CA, USA) as per the manufacturer's protocol.

\section{Methylation Array}

Values representing the methylation status (DNAm values) of 485577 cytosines within cytosine-phosphate-guanine $(\mathrm{CpG})$ loci were determined using Illumina Infinium HumanMethylation450 Beadchip Array (Illumina, San Diego, CA, USA) as per manufacturer's protocol.

Table I Characteristics of Cohorts for Measure of DNAm

\begin{tabular}{|c|c|c|c|c|c|c|}
\hline \multirow[b]{2}{*}{ Cohort } & \multicolumn{2}{|c|}{ Initial group of subjects } & \multicolumn{2}{|c|}{ DNAm array } & \multicolumn{2}{|c|}{ Melt curve analysis } \\
\hline & Younger & Older & Younger & Older & Younger & Older \\
\hline Sex & $17 \mathrm{M}, 5 \mathrm{~F}$ & $18 \mathrm{M}, 4 \mathrm{~F}$ & $15 \mathrm{M}, 5 \mathrm{~F}$ & $15 \mathrm{M}, 4 \mathrm{~F}$ & $13 \mathrm{M}, 5 \mathrm{~F}$ & $12 \mathrm{M}, 4 \mathrm{~F}$ \\
\hline Race & $20 \mathrm{~W}, 2 \mathrm{~B}$ & $19 \mathrm{~W}, 3 \mathrm{~B}$ & $20 \mathrm{~W}$ & $19 \mathrm{~W}$ & $18 \mathrm{~W}$ & $16 \mathrm{~W}$ \\
\hline Brain $\mathrm{pH}$ & $6.67 \pm 0.05$ & $6.76 \pm 0.05$ & $6.65 \pm 0.05$ & $6.78 \pm 0.05$ & $6.65 \pm 0.05$ & $6.76 \pm 0.06$ \\
\hline RIN & $8.19 \pm 0.13$ & $7.87 \pm 0.17$ & $8.22 \pm 0.14$ & $8.07 \pm 0.15$ & $8.29 \pm 0.14$ & $7.93 \pm 0.15$ \\
\hline BDNF Expression ${ }^{\mathrm{a}}$ & $25.22 \pm 0.66$ & $19.65 \pm 0.77$ & $24.91 \pm 0.68$ & $20.06 \pm 0.79$ & $24.72 \pm 0.68$ & $19.65 \pm 0.90$ \\
\hline SST Expression ${ }^{\mathrm{a}}$ & $682.27 \pm 23.17$ & $379.28 \pm 34.96$ & $675.37 \pm 24.67$ & $385.97 \pm 38.34$ & $671.28 \pm 27.31$ & $380.20 \pm 41.28$ \\
\hline
\end{tabular}

Abbreviations: B, black; F, female; $M$, male; PMI, postmortem interval; RIN, RNA integrity number; $W$, white.

Data for continuous variables are presented as 'group average \pm SEM'.

aMicroarray signal intensities. 


\section{Data Preprocessing and Filtering}

Analyses of the data were performed using the 'R' software environment. DNAm values of the $\mathrm{CpG}$ loci analyzed were calculated as both the ratio of signal from a methylated probe relative to the sum of both methylated and unmethylated probes ( $\beta$-value) and the log2 transformation of the ratio of a methylated probe over the unmethylated probe ( $M$-value). Because the $\beta$-value is heteroscedastic at the extremes of its range, the $M$-value was used for analysis. Corresponding $\beta$-values were used in final data representations given their intuitive biological interpretation; that is, it corresponds roughly to the proportion of a particular $\mathrm{CpG}$ locus that is methylated in a sample.

Preprocessing of the data, including color adjustment, background correction, and quantile normalization, were performed using the BioConductor lumi package. Poor quality (1260 probes for which a reliable read could not be made in $\geqslant 80 \%$ of samples) and invariable (242 788 probes $M$-value SD cut point of 0.147$)$ probes were filtered out of the data set. Global DNAm pattern, as assessed by multidimensional scaling (Cox and Cox, 2001), was not changed by probe filtering but was highly dependent on sex (Supplementary Figure S1A and B), an effect that disappeared upon filtering out probes (10 815 probes) associated with the X and Y chromosomes (Supplementary Figure S1C). After filtering, data from 230875 probes remained for downstream analysis. Global DNAm pattern was also highly dependent on race (Supplementary Figure S1C and D), and as only 5 of the 44 subjects were black, they were removed from this analysis. Twenty and 19 individuals remained in the younger and older cohorts, respectively (Table 1). Initially, only those probes associated with BDNF (26) and SST (9) based on UCSC build hg19 were used for downstream analysis. Subsequent analysis included a larger group of BDNF- and GABA-related genes: NTRK2, GAD1, GAD2, CORT, NPY, PVALB, CCK, VIP, GABRA1, GABRA2, and GABRA5 (Tripp et al, 2012).

\section{Differentially Methylated Regions}

To detect regions of the genome in which $\mathrm{CpG}$ loci are differentially methylated between cohorts (ie, DMRs), DNAm values for each individual was smoothed (500-bp sliding window), and a two-tailed $t$-test of smoothed DNAm values for each $\mathrm{CpG}$ locus between cohorts was performed. DMRs were then defined by merging continuous CpG loci that were differentially methylated between cohorts and for which the differences were concordant with respect to direction of effect (ie, hypermethylated or hypomethylated) within a 1000-bp window.

\section{Melt Curve Analysis}

Melt curve analysis was performed based on the methods described in Smith et al (2009). Briefly, PCR primers specific for bisulfite-converted DNA were designed towards DMRs using MethPrimer (Li and Dahiya, 2002) and used only if they produced a single product and produced no dimers or non-specific signal when amplified without bisulfiteconverted gDNA (Figures 1a and 2a; Supplementary Table S2). Primers toward DMR5 could not be designed.
Bisulfite-converted gDNA ( 7.5 ng) was amplified in $15 \mu \mathrm{l}$ reactions $\left(0.3 \times\right.$ SYBR-green, $3 \mathrm{mM} \mathrm{MgCl}_{2}, 200 \mu \mathrm{m}$ dNTPs, $200 \mu \mathrm{m}$ primers, 0.5 unit Taq DNA polymerase). Amplification was performed on a Eppendorf Mastercycler realplex thermal cycler (Eppendorf, Hamburg, Germany). Following amplification, the PCR product was subjected to a double-strand DNA melting protocol in which temperature was ramped from 73 to $90{ }^{\circ} \mathrm{C}$, rising $0.4^{\circ} \mathrm{C}-0.5^{\circ} \mathrm{C}$ at each step ( 7 s/step).

Relative methylation of the genomic region amplified by each primer set was determined by analyzing the melt curve of the PCR product. The temperature at which half the amplicons melted $\left(T_{50}\right)$ was calculated using fluorescence values measured during the DNA melting protocol. When comparing the same primer product between cohorts, a higher $T_{50}$ indicates increased DNAm.

\section{Bisulfite Pyrosequencing}

Pyrosequencing analysis of bisulfite-converted genomic DNA was performed by EpigenDx Inc. (Worcester, MA, USA) in regions close to and partially overlapping with the SST DMRs. The following primer sets were used: ADS3300, ADS3299, ADS3048, and ADS3049.

\section{RESULTS}

\section{BDNF is Hypermethylated in the OFC of Older Individuals}

Five BDNF DMRs between the cohorts were identified using the analysis described above. Separate DMRs were identified in the following regions: (1) upstream of exon I (DMR1), (2) exon II (DMR2), (3) upstream of exon IV (DMR3), (4) exon VI (DMR 4), and (5) downstream of exon VII (DMR5; Figure 1a and b; Table 2). All $10 \mathrm{CpG}$ loci that comprised the BDNF DMRs were hypermethylated in the older cohort. DNAm at each of the BDNF DMRs correlates positively with age (Supplementary Table S3).

Melt curve analysis confirmed that the BDNF DMRs identified using the array data were hypermethylated as the PCR products obtained from DMR2, DMR3, and DMR4 had $T_{50}$ values which were significantly higher in the older cohort. The $T_{50}$ value for DMR1 showed a strong statistical trend towards being higher in the older cohort (Figure 1a and c; Table 2). DMR5 could not be confirmed due to the inability to design effective bisulfite-specific PCR primers and was not included in the additional analyses.

\section{DNAm Negatively Correlates with BDNF Expression}

DMR1 DNAm negatively correlated with BDNF expression $(r=-0.37, N=39, p<0.05)$ and DMR2 DNAm negatively correlated with BDNF expression $(r=-0.40, N=39, p<0.05)$. There was a statistical trend towards negative correlation between DMR3 DNAm and BDNF expression $(r=-0.24$, $N=39, p=0.07)$. DMR4 DNAm did not correlate with BDNF expression $(r=-0.19, N=39, p=0.13$ ) (Figure $1 \mathrm{~d}-\mathrm{g}$ ).

\section{SST is Hypermethylated in the OFC of Older Individuals}

Two DMRs were identified in SST between cohorts. DMR1 was located upstream of the SST transcriptional start site and 
a

i.

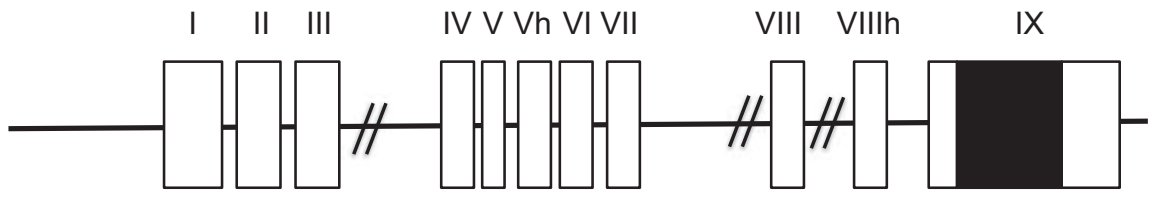

ii.

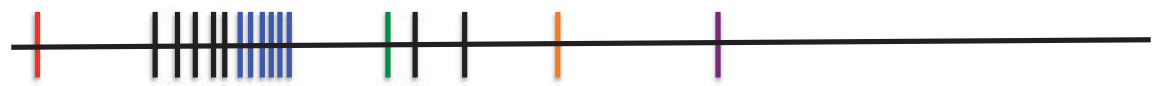

iii.

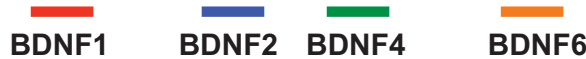

b

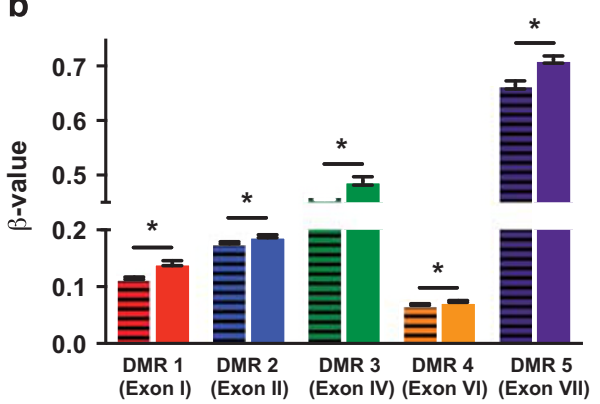

d
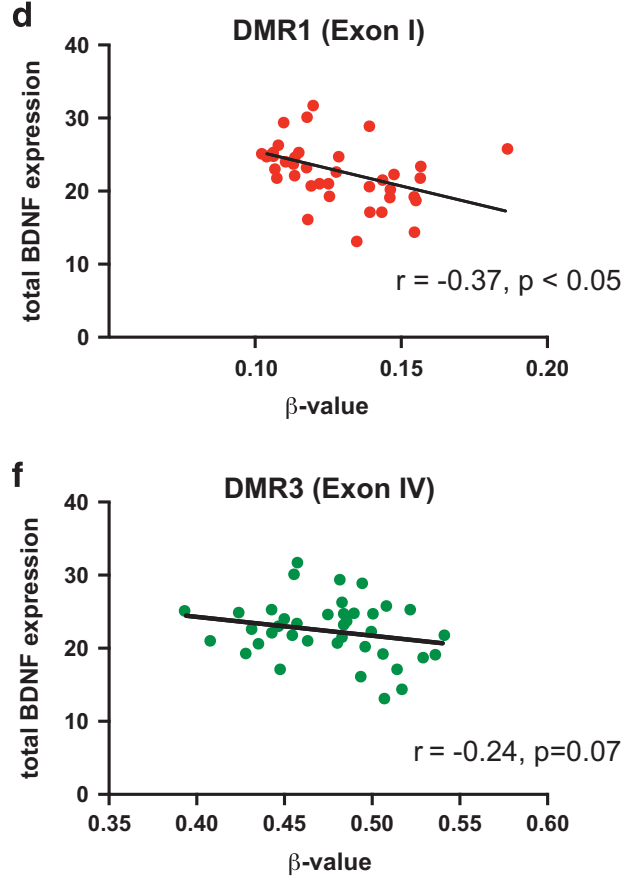

C
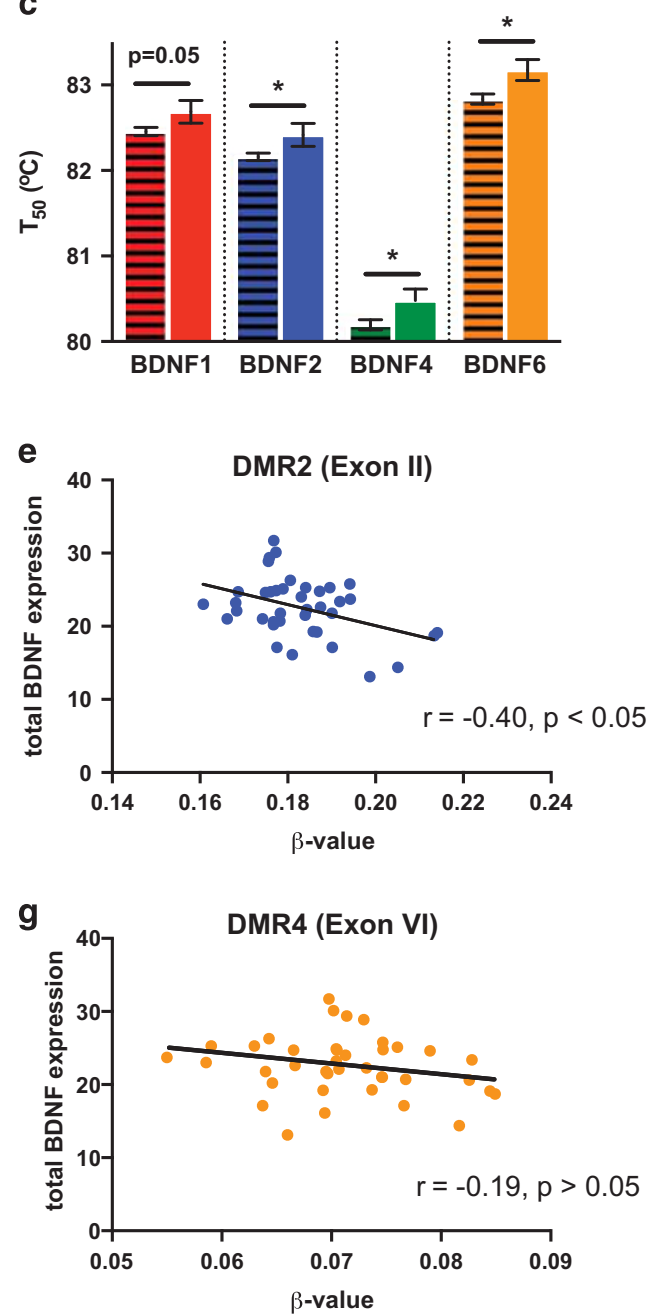

Figure I BDNF DNAm and gene expression. Throughout the figure, schematics/data associated with each of the differentially methylated regions (DMRs) are color coded: DMRI (red), DMR2 (blue), DMR3 (green), and DMR4 (orange). (a) (i) Schematic representation of BDNF gene structure. Exons are represented as white boxes and the introns as lines. The roman numerals indicate exon number. The black box represents the translated region. (ii) The positions of the CpG loci measured by Illumina Infinium HumanMethylation450 BeadChip Array and used in downstream analysis are marked by vertical lines. (iii) The horizontal lines represent the regions amplified by bisulfite-specific PCR primer sets (Supplementary Table SI). The names of the primer sets appear below the horizontal lines. (b) Average $\beta$-value of all the CpG loci within a DMR in the younger (horizontal stripes) and older (solid) cohorts. (c) Average $T_{50}$ of the amplification product produced by each of the primer sets in the younger (horizontal stripes) and older (solid) cohorts. ( $\mathrm{d}-\mathrm{g}$ ) Correlation between average $\beta$-value at each DMR and total BDNF expression. Error bars represent \pm SEM and $* p<0.05$.

CpG loci that comprised DMR2 were spread across the $5^{\prime}$ UTR, first exon, and intron (Figure 2a and b; Table 2). All eight of the CpG loci that comprised the DMRs were hypermethylated in the older cohort. DNAm at each of the
SST DMRs correlates positively with age (Supplementary Table S3).

Melt curve analysis supported the interpretation that SST is hypermethylated in the OFC of older individuals. Of the 
a

i.

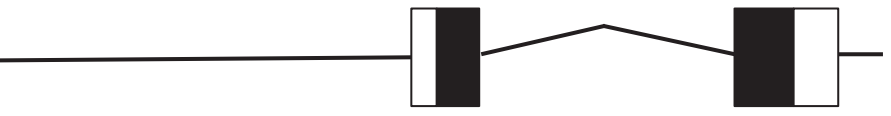

ii.

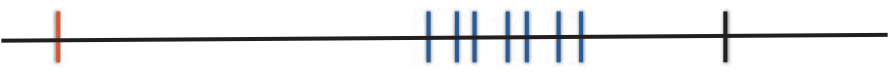

iii.
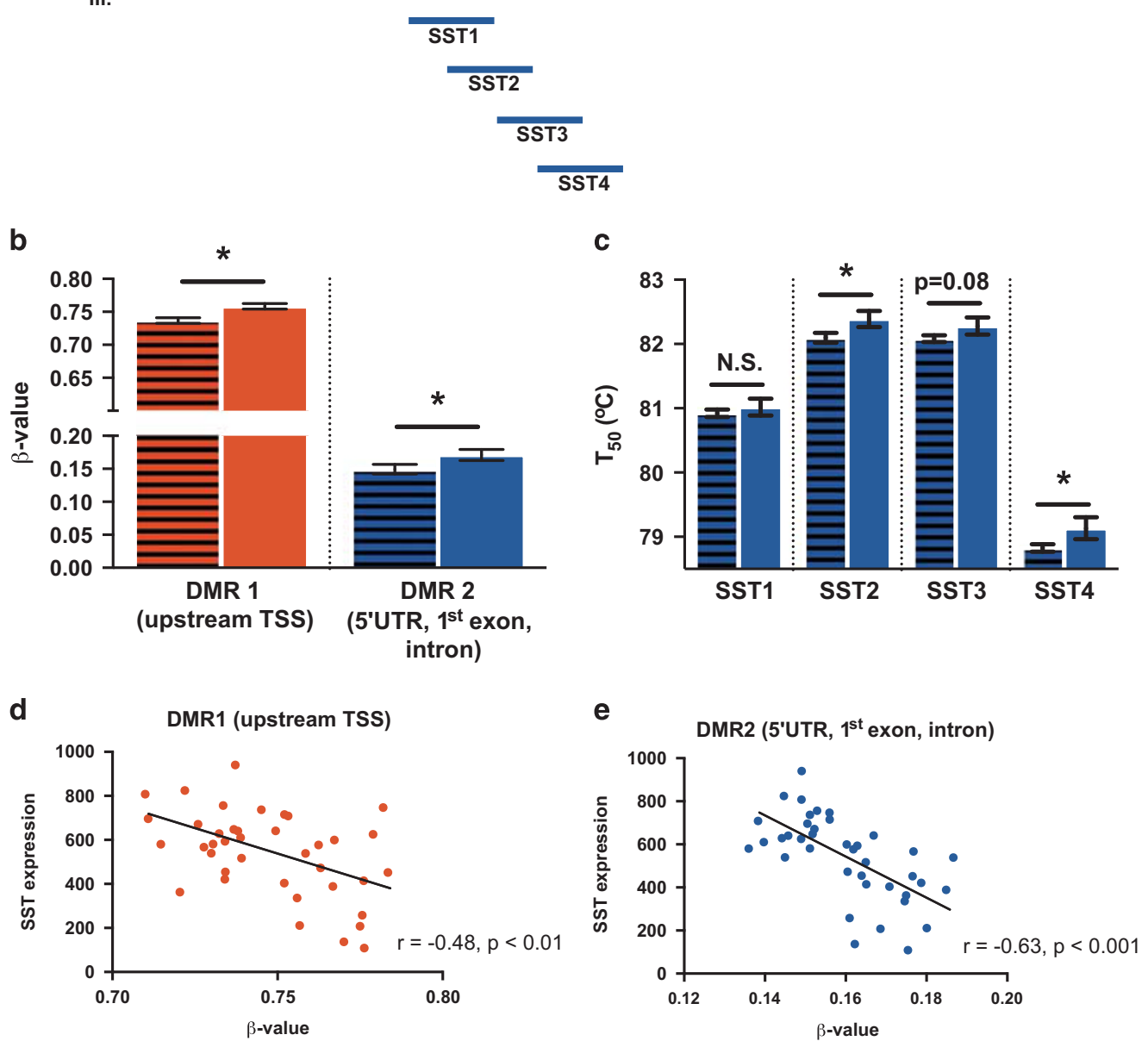

Figure 2 SST DNAm and gene expression. Throughout the figure, schematics/data associated with each of the differentially methylated regions (DMRs) are color coded: DMRI (red) and DMR2 (blue). (a) (i) Schematic representation of SST gene structure. Exons are represented as white boxes and the introns as lines. The black box represents the translated regions. (ii) The positions of the CpG loci measured by Illumina Infinium HumanMethylation450 BeadChip Array and included in downstream analysis are marked by vertical lines. (iii) The horizontal lines represent the regions amplified by bisulfite-specific PCR primer sets (Supplementary Table SI). The names of the primer sets appear below the horizontal lines. (b) Average $\beta$-value of all the CpG loci within a DMR in the younger (horizontal stripes) and older (solid) cohorts. (c) Average $T_{50}$ of the amplification product produced by each of the primer sets in the younger (horizontal stripes) and older (solid) cohorts. ( $\mathrm{d}$ and e) Correlation between average $\beta$-value at each DMR and total SST expression. Error bars represent \pm SEM and $* p<0.05$

PCR products produced by four different primer sets (SST1-4) directed towards SST, two (SST2 and SST4) had a significantly higher $T_{50}$ in the older cohort and a third (SST3) trended in this direction. The $T_{50}$ of SST1 did not differ between cohorts (Figure 2a and c; Table 2).

Bisulfite pyrosequencing of a region near and overlapping with the SST DMRs (chr3:187389514-187387808) further validated the results of the array and melt curve analysis. Specifically, 9 of the $14 \mathrm{CpG}$ sites measured by pyrosequencing in this region were significantly hypermethylated in the older cohort (Supplementary Table S4).

\section{DNAm Negatively Correlates with SST Expression}

DMR1 DNAm correlated negatively with the SST expression $(r=-0.48, n=39, p<0.01$; Figure $2 \mathrm{~d})$, and DMR2 DNAm correlated negatively with the SST expression $(r=-0.63$, $n=39, p<0.001$; Figure 2e).

\section{Age-Dependent Patterns of Decreased Expression and Hypermethylation Extends to other BDNF- and GABA-Related Genes}

As we have previously demonstrated a role for BDNF in regulating the expression of multiple GABA-related genes, 
Table 2 DNAm Array and Melt Curve Data for BDNF and SST DMRs

\begin{tabular}{|c|c|c|c|c|}
\hline \multirow{2}{*}{$\frac{\text { BDNF }}{\mathrm{DMR}}$} & \multicolumn{4}{|c|}{ DNAm array } \\
\hline & Associated exon/promoter & Younger cohort $(\beta$-value \pm SEM $)$ & Older cohort $(\beta$-value \pm SEM) & $p$-value \\
\hline 2 & $\|$ & $0.177 \pm 0.002$ & $0.189 \pm 0.003$ & $<0.01$ \\
\hline 3 & IV & $0.463 \pm 0.007$ & $0.489 \pm 0.008$ & $<0.05$ \\
\hline
\end{tabular}

Melt curve analysis

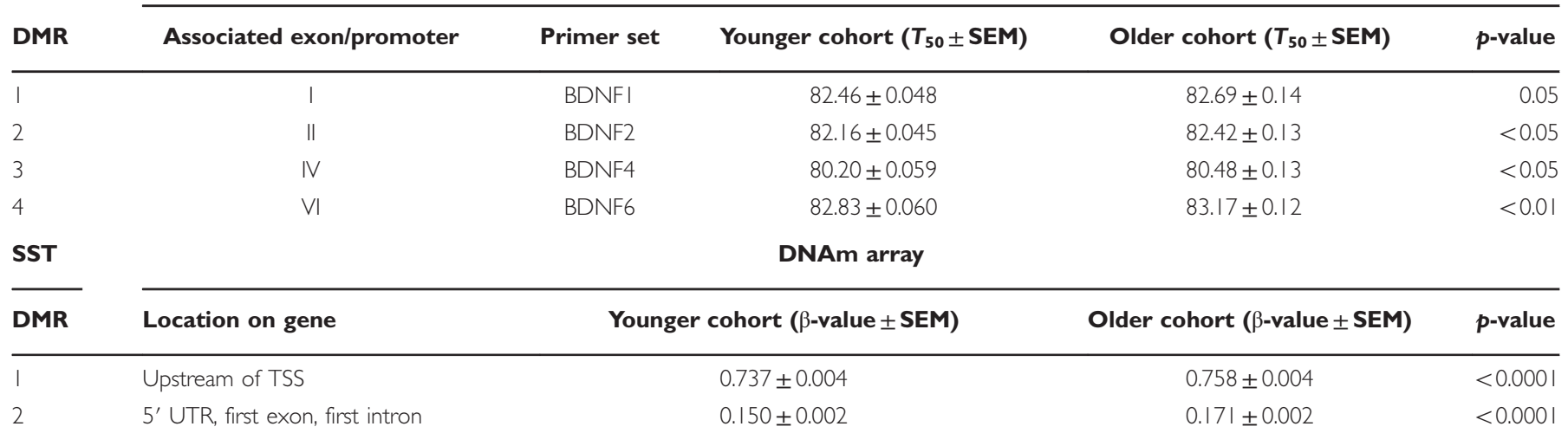

Melt curve analysis

\begin{tabular}{|c|c|c|c|c|c|}
\hline DMR & Location on gene & Primer set & Younger cohort $\left(T_{50} \pm\right.$ SEM) & Older cohort ( $T_{50} \pm$ SEM) & $p$-value \\
\hline 2 & Upstream of TSS, $5^{\prime} \cup T R$, first exon & SST2 & $82.10 \pm 0.08$ & $82.39 \pm 0.13$ & $<0.05$ \\
\hline 2 & First exon, first intron & SST4 & $78.83 \pm 0.06$ & $79.13 \pm 0.17$ & $<0.05$ \\
\hline
\end{tabular}

we explored the effect of aging on the expression and DNAm of a larger group of genes, including the BDNF receptor NTKR2 and GABA-related genes, for which we have previously demonstrated that expression is dependent, to variable degrees, on BDNF expression. This includes GAD1, GAD2, CORT, NPY, PVALB, CCK, VIP, GABRA1, GABRA2, and GABRA5 (Tripp et al, 2012) (Table 3). Of this larger group of BDNF- and GABA-related genes, only PVALB and GABRA2 were not differentially expressed between the younger and older cohorts, though there was a trend towards decreased PVALB expression in the older cohort $(p=0.09)$. Of those that were differentially expressed, NTKR2 was the only gene for which expression was significantly increased in the older cohort, and the expression of all others (GAD1, GAD2, CORT, NPY, GABRA1, and GABRA5) was significantly lower in the older cohort. All of the genes that were differentially expressed between cohorts were significantly hypermethylated in the older cohort, and a significant negative correlation between methylation and gene expression existed for all except NTKR2 and VIP. Of those that were not differentially methylated between cohorts, PVALB was significantly hypermethylated in the older cohort, and a significant negative correlation between methylation and expression existed. GABRA2 was not differentially methylated between groups, and there was no significant relationship between methylation and expression (Table 3).

\section{DISCUSSION}

DNA methylation (DNAm) changes in the brain with aging, and evidence suggests that these changes might underlie agerelated changes in gene expression (Fraga and Esteller, 2007). In this study, we demonstrate that two genes for which low expression is associated with brain aging and brain disorders, BDNF and SST, are hypermethylated in the OFC as part of normal brain aging. We also demonstrate that DNAm in these genes is negatively correlated with their expression. Finally, we found that most genes in a larger set of BDNFand GABA-related genes also displayed decreased expression and hypermethylation with aging and significant negative correlations between these two measures. These results provide evidence for DNAm as a putative mechanism for age-related changes in gene expression in the brain.

Our finding that BDNF is hypermethylated in the older cohort and that BDNF DNAm negatively correlates with its 
Table 3 Most BDNF- and GABA-Related Genes Examined Exhibit Decreased Expression and Increased Methylation in the Older Cohort, Negative Correlation Between Expression and Methylation

\begin{tabular}{|c|c|c|c|c|}
\hline $\begin{array}{l}\text { Functional } \\
\text { Group }\end{array}$ & Genes & $\begin{array}{c}\text { Age expression } \\
\text { (older in \% of } \\
\text { younger) *, } \\
\mathbf{p}<0.01\end{array}$ & $\begin{array}{c}\text { DMRs } \\
\text { (all } \\
p<0.05 \text { ) }\end{array}$ & $\begin{array}{l}\text { Expression/ } \\
\text { Methylation } \\
\mathbf{R} *, \mathbf{p}<0.05\end{array}$ \\
\hline BDNF & BDNF & $* 80$ & DMRI & $*-0.37$ \\
\hline \multirow[t]{4}{*}{ Signaling } & & & DMR2 & $*-0.4$ \\
\hline & & & DMR3 & -0.24 \\
\hline & & & DMR4 & -0.19 \\
\hline & NTRK2 & $* 107.2$ & DMRI & 0.15 \\
\hline GABA & GADI & *85.4 & DMRI & $*-0.41$ \\
\hline Synthesizing & & & DMR2 & $*-0.45$ \\
\hline \multirow[t]{4}{*}{ Enzymes } & & & DMR3 & -0.31 \\
\hline & & & DMR4 & -0.02 \\
\hline & GAD2 & $* 77.2$ & DMRI & $*-0.36$ \\
\hline & & & DMR2 & -0.3 \\
\hline GABA-ergic & SST & *58.1 & DMR I & $*-0.048$ \\
\hline Interneuron & & & DMR2 & $*-0.63$ \\
\hline \multirow[t]{7}{*}{ Markers } & CORT & * 82.4 & DMR I & $*-0.53$ \\
\hline & & & DMR 2 & $*-0.51$ \\
\hline & NPY & *83. 1 & DMR I & $*-0.09$ \\
\hline & PVALB & $85.1 \quad(p=0.09)$ & DMRI & -0.22 \\
\hline & & & DMR2 & $*-0.48$ \\
\hline & CCK & * 90.5 & DMRI & $*-0.61$ \\
\hline & VIP & *70.5 & DMRI & 0.19 \\
\hline GABA & GABRAI & *87.6 & DMR I & -0.22 \\
\hline \multirow[t]{8}{*}{ Receptors } & & & DMR 2 & $*-0.37$ \\
\hline & GABRA5 & *75.4 & DMRI & $*-0.52$ \\
\hline & & & DMR2 & $*-0.34$ \\
\hline & & & DMR3 & $*-0.57$ \\
\hline & & & DMR4 & -0.12 \\
\hline & & & DMR5 & $*-0.53$ \\
\hline & & & DMR6 & $*-0.37$ \\
\hline & & & DMR7 & $*-0.42$ \\
\hline
\end{tabular}

Abbreviations: $r$, Pearson correlation coefficient; $\mathrm{SE}_{n}$ standard error of $r$, TSS, transcriptional start site; UTR, untranslated region.

${ }^{\text {a }}$ Statistically significant $r$ value.

DMRs that fall within the $5^{\prime}$ untranslated region and/or within I 500 bp upstream of the transcriptional start site of the associated gene are grey shaded.

expression supports our prediction and a previous study that showed BDNF hypermethylation and decreased BDNF protein level in postmortem prefrontal cortex of older individuals (Keleshian et al, 2013). This previous study, however, only measured DNAm of the CpG Island associated with BDNF exon I. We were able to measure DNAm at $26 \mathrm{CpG}$ loci distributed throughout multiple BDNF exons and identified four BDNF DMRs between cohorts localized to, or near, BDNF exons I, II, IV, and VI. DNAm at all BDNF DMRs except for the one associated with BDNF exon VI was negatively correlated with BDNF expression.

Human BDNF has a complex gene structure consisting of eight untranslated exons and a single coding exon (exon IX).
BDNF expression is spatiotemporally regulated by these different exons (Pruunsild et al, 2007). Much evidence demonstrates a role for DNAm in the regulation of BDNF expression, and studies of the rodent Bdnf gene, which is similar in structure to the human BDNF gene, suggest that some exons are preferentially targeted by DNAm, most notably exons I and IV, in response to environmental stimuli (Ikegame et al, 2013). Human BDNF exons I and IV have also been found to be abnormally methylated in the brains of individuals with psychiatric disorders. For example, in postmortem frontal cortex of individuals with bipolar disorder and Alzheimer's disease, BDNF exon I is hypermethylated (Rao et al, 2012) and a significant increase in DNAm at four CpG sites within the BDNF IV exon was reported in Wernicke's area of suicide completers (Keller et al, 2010). Our results demonstrating that two of the three aging BDNF DMRs that significantly correlate with BDNF expression are localized to BDNF exons I and IV are consistent with the observation that these exons are targets of DNAm. Other BDNF exons are less consistently shown to be a target for DNAm in response to environmental stimuli or pathological state. That only a subset of BDNF exons appears to be targeted for DNAm with brain aging is consistent with different BDNF exons having different spatiotemporal distributions, functions, and regulation (Pruunsild et al, 2007).

Contrary to our prediction, SST was hypermethylated in the older cohort. In fact, eight of the nine CpG loci studied in SST belonged to one of the two SST DMRs. DNAm at both SST DMRs was negatively correlated with SST expression, and these correlations were somewhat stronger for SST than for BDNF. The fact that both BDNF and SST are hypermethylated in the older cohort and that DNAm of each gene is inversely correlated with its expression suggests that SST expression is subject to regulation by DNAm in addition to its previously demonstrated regulation by BDNF itself (Guilloux et al, 2012; Tripp et al, 2012).

Hypermethylation of the SST promoter and decreased SST expression are early markers of gastrointestinal cancers (Goel, 2011), but the evidence for DNAm regulation of SST expression in the human brain is minimal. A recent small study of SST promoter methylation in postmortem prefrontal cortex from individuals with Alzheimer's disease and age-matched controls found that the SST promoter was not differentially methylated between groups despite SST expression levels being significantly lower in the group with Alzheimer's disease. This study did find a moderate increase, although not statistically significant $(p=0.08)$, in DNAm of the CpG island associated with SST during aging (Grosser et al, 2014). The SST promoter was also one of the 273 gene promoters hypermethylated in the hippocampi of suicide completers (Labonte et al, 2013). Our findings are the first to associate changes in DNAm with changes in SST expression using multiple measures of DNAm in a large sample of human postmortem brains.

When correlating DNAm at individual SST CpG loci with SST expression, we find that two CpG loci in the intron correlate somewhat more strongly with SST expression than the other CpG loci, thus suggesting that this region may be particularly important to the regulation of age-related decreases in SST expression by DNAm (Supplementary Table S3). Of note, these same two CpG loci were included 
among the $71 \mathrm{CpG}$ loci used in a study to build a quantitative model of human aging from whole blood (Hannum et al, 2013), perhaps suggesting that peripheral whole blood could be used as a surrogate for brain tissue when studying effects of age on SST methylation.

The observation that most genes in a set of BDNF- and GABA-related genes that we assessed exhibited similar agerelated changes in DNAm and correlation with gene expression suggests that DNAm may be a mechanism for regulation of gene expression for a large number of genes. The fact that this phenomenon does not seem to depend on the degree to which a gene's expression is dependent on BDNF levels further suggests that these are two independent mechanisms by which expression of BDNF- and GABArelated genes are regulated. Finally, the observation that DNAm is highly correlated at DMRs within BDNF- and GABA-related genes as well as between BDNF- and GABArelated genes in some, but not all, cases (Supplementary Table S6) suggests that DNAm in these regions and thus expression of particular BDNF- and GABA-related genes and transcripts regulated in a coordinated way in normal brain aging, perhaps by a common upstream regulator. This observation, however, is at odds with our initial hypothesis that low SST expression (and by extension, other GABArelated genes for which expression is dependent on BDNF levels) is downstream of low BDNF expression. If low SST expression (or GABA-related gene expression) were indeed only dependent on low BDNF expression, we would not expect to see any significant correlation of DNAm between the DMRs in the two genes.

Though robust and concordant across multiple CpG loci in the same gene, the DNAm differences between cohorts were subtle (1-5\%). These small DNAm effect sizes are in line with those previously reported for the effect of aging, brain disorders, and environmental factors on DNAm (Fraga and Esteller, 2007; Rao et al, 2012). It may be that these small changes are functionally meaningful to the few neurons in which they occur; indeed, small changes in DNAm have been demonstrated to lead to measurable functional outcomes (Lim et al, 2012). Alternatively, it may be that the effect size would be much larger if DNAm was measured only in those cells in which BDNF and SST are expressed but addressing this will require obtaining more homogenous cell/nuclei populations from the postmortem brain tissue by laser capture microscopy (Bernard et al, 2011), fluorescent-activated nuclei sorting (Jiang et al, 2008), or similar methods.

At least four mechanisms that broadly drive age-related changes in DNAm may contribute to our observations. First, genetic variation may contribute to age-related changes in DNAm. Of note, DNAm at BDNF and SST CpG sites that exhibited age-related changes in DNAm were not significantly associated with genetic variance within $1 \mathrm{Mb}$ of the site (data not shown). Second, exposure to certain environmental factors activates biological programs that give rise to consistent and predictable changes in DNAm. Exposure to differing nutrients, substances of abuse, types of stress, and therapeutic interventions are just some of the environmental factors that have been shown to affect DNAm (Zhang and Meaney, 2010). This mechanism would lead to age-related differences in DNAm that depend on the accumulation of a unique set of life experiences. Third, spontaneous change in DNAm contributes to age-related changes in DNAm. Such spontaneous changes might arise from errors in the processes that maintain DNAm status at a particular site in the genome. The rates of spontaneous changes may differ depending on cell type. For example, one might imagine continuously dividing glial cells to have higher rates of spontaneous change than largely postmitotic neurons. Fourth, a 'DNAm clock' mechanism may contribute to age-related changes in DNAm. Support for this hypothesis comes from the observation that DNAm at some CpG loci changes so predictably with age that they can be used to predict age (Bocklandt et al, 2011). Understanding the DNAm clock may be particularly valuable for extending lifespan and developing therapeutics for brain disorders thought to be disorders of accelerated aging, such as major depressive disorder (Wolkowitz et al, 2011), bipolar disorder (Rizzo et al, 2014), and schizophrenia (Kirkpatrick et al, 2008).

The proximal upstream mechanisms leading to hypermethylation of BDNF and SST with brain aging are not understood. A putative mechanism is suggested by the recent observation that aging is associated with a significant induction of the transcriptional repressor element-1 silencing factor (REST) in neuronal nuclei and increased binding of REST to target genes, such as BDNF and SST (Lu et al, 2014). Once bound to a target gene, REST creates a repressive chromatin structure and inhibits target gene expression through recruitment of co-repressors, including histone deacetylases, enzymes known to regulate DNAm (Vaissiere et al, 2008). To explore whether our findings could be related to REST binding, we searched the genomic coordinates of each of the BDNF and SST DMRs in UCSC Genome Browser (build hg19) with the Encyclopedia of DNA elements (ENCODE) Transcription Factor Track activated. REST was found to have signal strength indicative of high occupancy at or near the DMRs associated with BDNF exons I and II and the SST DMR 2 in multiple transformed cell lines, including many of neural and glial origin (eg, U87, SK-N-SH, and PFSK-1), but notably there is no track corresponding to primary neurons. REST occupancy was not associated with the DMRs associated with BDNF exon IV or SST DMR 1 (data not shown). Similarly, the downstream mechanisms by which hypermethylation of BDNF and SST might cause decreased BDNF and SST expression are unknown. Generally, gene expression is proximally regulated through spatiotemporal interactions between transcription factors and their cognate recognition sites on genomic DNA. How DNAm at a particular CpG locus affects these interactions is likely relevant to expression of the gene with which it is associated. One can imagine how hypermethylation of BDNF and SST might lead to decreased expression by (1) preventing an interaction between an activating transcription factor and its recognition site, or (2) promoting an interaction between a repressive transcription factor and its recognition site, within the BDNF and/or SST genes (Baubec and Schubeler, 2014).

The present study raises the possibility that DNAm functions as a regulator of gene expression observed in brain disorders in which low expression of BDNF and SST have been found. It also supports a role for DNAm as a putative mechanism for mediating the age-by-disease changes in gene expression that we have previously hypothesized to contribute to the late-life brain disorders. 


\section{Notes and Limitations}

Some limitations of our study are inherent to postmortem brain studies. For example, the findings are only correlative and cannot reveal the mechanistic relationship between DNAm and gene expression in normal brain aging. Also, tissue quality and preservation of biological macromolecules and their modifications are always of concern in postmortem brain studies. It is notable that parameters related to tissue quality (PMI, pH, RIN) are similar in both cohorts. Because we chose cohorts such that the older cohort would be enriched in individuals with particularly low levels of BDNF and SST expression, the ability to generalize our findings to individuals with intermediate expression levels may be limited. Additional studies will be necessary to confirm the association of DNA hypermethylation with age-related decreases in BDNF and SST expression in brain regions other than the OFC. Another limitation, a product of the technology used, is the fact that we only studied a subset of the CpG loci associated with BDNF and SST. Finally, DMRs associated with BDNF exons I, IV, VI, and the upstream region of SST are represented by a single CpG locus, thus raising the question of whether it is DNAm at this particular locus that is important for gene expression or whether this particular locus is simply one of the many loci in that region of the genome that is similarly methylated. The melting curve experiments provide some insight into this question, as the $T_{50}$ of the PCR product amplified from the bisulfiteconverted genome reflects the average DNAm of all the CpG loci in that region of the genome and most of the DMRs had higher $T_{50}$ in the older group, a finding that suggests that multiple CpG loci in the region are hypermethylated in the older group.

\section{FUNDING AND DISCLOSURE}

This work was supported by National Institute of Mental Health grants R01MH077159 and R01MH093723 (to ES) and University of Pittsburgh Department of Psychiatry David J. Kupfer Residency Research Fellowship (BCM). DAL currently receives investigator-initiated research support from Pfizer and in 2012-2014 served as a consultant in the areas of target identification and validation and new compound development to Autifony, Bristol-Myers Squibb, Concert Pharmaceuticals, and Sunovion. The funding agencies had no role in the study design, data collection and analysis, decision to publish, or preparation of the manuscript. The authors declare no conflict of interest.

\section{REFERENCES}

Autry AE, Monteggia LM (2012). Brain-derived neurotrophic factor and neuropsychiatric disorders. Pharmacol Rev 64: 238-258.

Baubec T, Schubeler D (2014). Genomic patterns and context specific interpretation of DNA methylation. Curr Opin Genet Dev 25C: $85-92$.

Bernard R, Burke S, Kerman IA (2011). Region-specific in situ hybridization-guided laser-capture microdissection on postmortem human brain tissue coupled with gene expression quantification. Methods Mol Biol 755: 345-361.

Bocklandt S, Lin W, Sehl ME, Sanchez FJ, Sinsheimer JS, Horvath S et al (2011). Epigenetic predictor of age. PloS one 6: e14821.
Cox TFC, Cox MAA (2001). Multidimensional Scaling. Chapman and Hall.

Erraji-Benchekroun L, Underwood MD, Arango V, Galfalvy H, Pavlidis P, Smyrniotopoulos P et al (2005). Molecular aging in human prefrontal cortex is selective and continuous throughout adult life. Biol Psychiatry 57: 549-558.

Fraga MF, Esteller M (2007). Epigenetics and aging: the targets and the marks. Trends Genet 23: 413-418.

Glorioso C, Oh S, Douillard GG, Sibille E (2011). Brain molecular aging, promotion of neurological disease and modulation by sirtuin 5 longevity gene polymorphism. Neurobiol Dis 41: 279-290.

Goel A (2011). Somatostatin methylation as a biomarker for gastric cancer: ready for 'prime time' or for further validation? Dig Dis Sci 56: 1-3.

Grosser C, Neumann L, Horsthemke B, Zeschnigk M, van de Nes J (2014). Methylation analysis of SST and SSTR4 promoters in the neocortex of Alzheimer's disease patients. Neurosci Lett 566: 241-246.

Guilloux JP, Douillard-Guilloux G, Kota R, Wang X, Gardier AM, Martinowich $\mathrm{K}$ et al (2012). Molecular evidence for BDNF- and GABA-related dysfunctions in the amygdala of female subjects with major depression. Mol Psychiatry 17: 1130-1142.

Hannum G, Guinney J, Zhao L, Zhang L, Hughes G, Sadda S et al (2013). Genome-wide methylation profiles reveal quantitative views of human aging rates. Mol Cell 49: 359-367.

Hayashi M, Yamashita A, Shimizu K (1997). Somatostatin and brain-derived neurotrophic factor mRNA expression in the primate brain: decreased levels of mRNAs during aging. Brain Res 749: 283-289.

Ikegame T, Bundo M, Murata Y, Kasai K, Kato T, Iwamoto K (2013). DNA methylation of the BDNF gene and its relevance to psychiatric disorders. J Hum Genet 58: 434-438.

Jiang Y, Matevossian A, Huang HS, Straubhaar J, Akbarian S (2008). Isolation of neuronal chromatin from brain tissue. BMC Neurosci 9: 42.

Keleshian VL, Modi HR, Rapoport SI, Rao JS (2013). Aging is associated with altered inflammatory, arachidonic acid cascade, and synaptic markers, influenced by epigenetic modifications, in the human frontal cortex. J Neurochem 125: 63-73.

Keller S, Sarchiapone M, Zarrilli F, Videtic A, Ferraro A, Carli V et al (2010). Increased BDNF promoter methylation in the Wernicke area of suicide subjects. Arch Gen Psychiatry 67: 258-267.

Kirkpatrick B, Messias E, Harvey PD, Fernandez-Egea E, Bowie CR (2008). Is schizophrenia a syndrome of accelerated aging? Schizophr Bull 34: 1024-1032.

Labonte B, Suderman M, Maussion G, Lopez JP, Navarro-Sanchez L, Yerko V et al (2013). Genome-wide methylation changes in the brains of suicide completers. Am J Psychiatry 170: 511-520.

Li L, Dahiya R (2002). MethPrimer: designing primers for methylation PCRs. Bioinformatics 18: 1427-1431.

Lim AL, Ng S, Leow SC, Choo R, Ito M, Chan YH et al (2012). Epigenetic state and expression of imprinted genes in umbilical cord correlates with growth parameters in human pregnancy. J Med Genet 49: 689-697.

Lin LC, Sibille E (2013). Reduced brain somatostatin in mood disorders: a common pathophysiological substrate and drug target? Front Pharmacol 4: 110.

Lu T, Aron L, Zullo J, Pan Y, Kim H, Chen Y et al (2014). REST and stress resistance in ageing and Alzheimer's disease. Nature 507: 448-454.

McKinney BC, Oh H, Sibille E (2012a). Age-by-disease biological interactions: implications for late-life depression. Front Genet 3: 237. 
McKinney BC, Sibille E (2012b). The age-by-disease interaction hypothesis of late-life depression. Am J Geriatr Psychiatry 21: 418-432.

Pruunsild P, Kazantseva A, Aid T, Palm K, Timmusk T (2007). Dissecting the human BDNF locus: bidirectional transcription, complex splicing, and multiple promoters. Genomics 90: 397-406.

Rao JS, Keleshian VL, Klein S, Rapoport SI (2012). Epigenetic modifications in frontal cortex from Alzheimer's disease and bipolar disorder patients. Transl Psychiatry 2: e132.

Rizzo LB, Costa LG, Mansur RB, Swardfager W, Belangero SI, Grassi-Oliveira R et al (2014). The theory of bipolar disorder as an illness of accelerated aging: implications for clinical care and research. Neurosci Biobehav Rev 42: 157-169.

Seney ML, Chang LC, Oh H, Wang X, Tseng GC, Lewis DA et al (2013). The role of genetic sex in affect regulation and expression of GABA-related genes across species. Front Psychiatry 4: 104.

Smith E, Jones ME, Drew PA (2009). Quantitation of DNA methylation by melt curve analysis. BMC Cancer 9: 123 .

Tapia-Arancibia L, Aliaga E, Silhol M, Arancibia S (2008). New insights into brain BDNF function in normal aging and Alzheimer disease. Brain Res Rev 59: 201-220.
Tripp A, Kota RS, Lewis DA, Sibille E (2011). Reduced somatostatin in subgenual anterior cingulate cortex in major depression. Neurobiol Dis 42: 116-124.

Tripp A, Oh H, Guilloux JP, Martinowich K, Lewis DA, Sibille E (2012). Brain-derived neurotrophic factor signaling and subgenual anterior cingulate cortex dysfunction in major depressive disorder. Am J Psychiatry 169: 1194-1202.

Vaissiere T, Sawan C, Herceg Z (2008). Epigenetic interplay between histone modifications and DNA methylation in gene silencing. Mutat Res 659: 40-48.

Webster MJ, Weickert CS, Herman MM, Kleinman JE (2002). BDNF mRNA expression during postnatal development, maturation and aging of the human prefrontal cortex. Brain Res Dev Brain Res 139: 139-150.

Wolkowitz OM, Reus VI, Mellon SH (2011). Of sound mind and body: depression, disease, and accelerated aging. Dialogues Clin Neurosci 13: 25-39.

Zhang TY, Meaney MJ (2010). Epigenetics and the environmental regulation of the genome and its function. Annu Rev Psychol 61: 439-466, C431-433.

Zuccato C, Cattaneo E (2009). Brain-derived neurotrophic factor in neurodegenerative diseases. Nat Rev Neurol 5: 311-322. 